\title{
Synthesis of Relativistic Wave Equations: The Noninteracting Case
}

\author{
Andrzej Okniński \\ Physics Division, Politechnika Świętokrzyska, Al. 1000-lecia PP 7, 25-314 Kielce, Poland \\ Correspondence should be addressed to Andrzej Okniński; fizao@tu.kielce.pl
}

Received 19 August 2015; Accepted 19 October 2015

Academic Editor: Ming Mei

Copyright ( 2015 Andrzej Okniński. This is an open access article distributed under the Creative Commons Attribution License, which permits unrestricted use, distribution, and reproduction in any medium, provided the original work is properly cited.

\begin{abstract}
We study internal structure of the Duffin-Kemmer-Petiau equations for spin 0 and spin 1 mesons. We show that in the noninteracting
\end{abstract} case full covariant solutions of the $s=0$ and $s=1$ DKP equations are generalized solutions of the Dirac equation.

\section{Introduction}

The Duffin-Kemmer-Petiau (DKP) equations [1-3], describing spin 0 and spin 1 mesons, are becoming increasingly useful due to their applications to problems in particle and nuclear physics [4-13]. It is well known that the DKP equations contain redundant components since only $2(2 s+$ 1) components are needed to describe free spin $s$ particles with nonzero rest masses $[14,15]$ while $s=0$ and $s=1$ DKP equations contain 5 and 10 components, respectively. The presence of redundant components in DKP equations leads for some interactions to such nonphysical effects as superluminal velocities $[16,17]$ (see also [18-20] for $s=3 / 2,2$ cases). On the other hand, physically acceptable equations for arbitrary spin can be obtained by removing redundant components with use of additional covariant condition [14, 15]. It seems that presence of redundant components means that the DKP equations have internal structure.

The motivation of this work is our recent discovery that solutions of subequations of the $s=0$ and $s=1 \mathrm{DKP}$ equations fulfill the Dirac equation [21, 22]. On the other hand, these solutions do not contain all spinor components and are thus noncovariant solutions of covariant equations. We studied this problem in $[23,24]$. In the present work, we show that, in the free case, full covariant solutions of the $s=0$ and $s=1 \mathrm{DKP}$ equations are generalized solutions of the Dirac equation. This finding may provide a basis for a synthesis of covariant particle equations, alternative to the classical Foldy programme [25].
The paper is organized as follows. In Section 2 the Dirac equation and the Duffin-Kemmer-Petiau equations for $s=0$ and $s=1$ are described. It is shown in Section 3 that in the noninteracting case solutions of these DKP equations are generalized matrix solutions of the Dirac equation. We discuss our findings in the last section.

\section{Relativistic Wave Equations}

In what follows we use conventions and definitions introduced in [22]. The Dirac equation, describing spin $1 / 2$ elementary particles, is

$$
\gamma_{\mu} p^{\mu} \Psi=m \Psi,
$$

where $\gamma^{\mu}$ are $4 \times 4$ matrices fulfilling [26, 27]

$$
\gamma^{\mu} \gamma^{\nu}+\gamma^{\nu} \gamma^{\mu}=2 g^{\mu \nu} I_{4 \times 4}
$$

where $g^{\mu \nu}=\operatorname{diag}(1,-1,-1,-1)$ and $I_{4 \times 4}$ is $4 \times 4$ unit matrix. In the spinor representation of the Dirac matrices we have $\Psi=\left(\xi^{A}, \eta_{\dot{B}}\right)^{T}[28]$, where $T$ denotes transposition of a matrix.

The DKP equations for spin 0 and spin 1 mesons are written as

$$
\beta_{\mu} p^{\mu} \Psi=m \Psi,
$$

where $\beta^{\mu}$ are $5 \times 5$ and $10 \times 10$ matrices, respectively, obeying the following commutation relations [1-3]:

$$
\beta^{\lambda} \beta^{\mu} \beta^{v}+\beta^{v} \beta^{\mu} \beta^{\lambda}=g^{\lambda \mu} \beta^{v}+g^{\nu \mu} \beta^{\lambda} .
$$


In the case of $s=0$ representation equation (3) can be written as

$$
\begin{aligned}
p^{\mu} \psi & =m \psi^{\mu}, \\
p_{\nu} \psi^{\nu} & =m \psi,
\end{aligned}
$$

with $\Psi$ in (3) defined as

$$
\Psi=\left(\psi^{\mu}, \psi\right)^{T}=\left(\psi^{0}, \psi^{1}, \psi^{2}, \psi^{3}, \psi\right)^{T} .
$$

In the case of $s=1$ (3) reduces to

$$
\begin{aligned}
p^{\mu} \psi^{\nu}-p^{\nu} \psi^{\mu} & =m \psi^{\mu \nu}, \\
p_{\mu} \psi^{\mu \nu} & =m \psi^{\nu},
\end{aligned}
$$

with $\Psi$ in (3) equalling

$$
\begin{aligned}
\Psi & =\left(\psi^{\mu \nu}, \psi^{\lambda}\right)^{T} \\
& =\left(\psi^{01}, \psi^{02}, \psi^{03}, \psi^{23}, \psi^{31}, \psi^{12}, \psi^{0}, \psi^{1}, \psi^{2}, \psi^{3}\right)^{T},
\end{aligned}
$$

where $\psi^{\lambda}$ are real and $\psi^{\mu \nu}$ are purely imaginary (alternatively, $-\partial^{\mu} \psi^{\nu}+\partial^{\nu} \psi^{\mu}=m \psi^{\mu \nu}$ and $\partial_{\mu} \psi^{\mu \nu}=m \psi^{\nu}$, where $\psi^{\lambda}$ and $\psi^{\mu \nu}$ are real). The $s=1$ condition, $p_{\nu} \psi^{\nu}=0$, follows from the second equation of (7) due to antisymmetry of tensor $\psi^{\mu \nu}$. Equations for spin 1 bosons (7) were first written by Proca [29].

\section{From the DKP Equations to}

\section{Generalized Solutions of the Dirac Equation}

3.1. Spin 0. Equations (5) can be written within spinor formalism as

$$
\begin{aligned}
p_{A \dot{B}} \psi & =m \psi_{A \dot{B}}, \\
p^{A \dot{B}} \psi_{A \dot{B}} & =2 m \psi .
\end{aligned}
$$

Splitting the last equation of (9), $p^{A \dot{B}} \psi_{A \dot{B}}=p^{1 \mathrm{i}} \psi_{1 \mathrm{i}}+$ $p^{1 \dot{2}} \psi_{1 \dot{2}}+p^{2 \dot{1}} \psi_{2 \dot{1}}+p^{2 \dot{2}} \psi_{2 \dot{2}}=2 m \psi$; we obtain two sets of equations involving components $\psi_{11}, \psi_{12}, \psi$ and $\psi_{21}, \psi_{22}, \psi$, respectively:

$$
\begin{aligned}
p_{1 \dot{1}} \psi & =m \psi_{1 \dot{1}}, \\
p_{1 \dot{2}} \psi & =m \psi_{1 \dot{2}}, \\
p^{1 \dot{1}} \psi_{1 \dot{1}}+p^{1 \dot{2}} \psi_{1 \dot{2}} & =m \psi, \\
p_{2 \dot{1}} \psi & =m \psi_{2 \dot{1}}, \\
p_{2 \dot{2}} \psi & =m \psi_{2 \dot{2}}, \\
p^{2 \dot{1}} \psi_{2 \dot{1}}+p^{2 \dot{2}} \psi_{2 \dot{2}} & =m \psi,
\end{aligned}
$$

each of which describes particle with mass $m$ (we check this substituting, e.g., $\psi_{11}$ and $\psi_{12}$ or $\psi_{21}$ and $\psi_{22}$, into the third equations). The splitting preserving $p_{\mu} p^{\mu} \psi=m^{2} \psi$ is possible due to spinor identities, $p_{1 i} p^{1 \mathrm{i}}+p_{21} p^{2 \mathrm{i}}=p_{\mu} p^{\mu}$ and $p_{1 \dot{2}} p^{1 \dot{2}}+$ $p_{2 \dot{2}} p^{2 \dot{2}}=p_{\mu} p^{\mu}$ (cf. [22]). Thus (10) and (11) are equivalent to DKP equations (9). We described similar equations in [21]. From each of (10) and (11) an identity follows:

$$
\begin{aligned}
& p_{1 \dot{2}} \psi_{1 \mathrm{i}}=p_{1 \mathrm{i}} \psi_{1 \dot{2}}, \\
& p_{2 \dot{2}} \psi_{2 \mathrm{i}}=p_{2 \mathrm{i}} \psi_{2 \dot{2}} .
\end{aligned}
$$

Equation (10) and the identity (12a), as well as (11) and the identity (12b), can be written in form of the Dirac equations:

$$
\begin{aligned}
& \left(\begin{array}{cccc}
0 & 0 & p_{1 \mathrm{i}} & p_{2 \mathrm{i}} \\
0 & 0 & p_{1 \dot{2}} & p_{2 \dot{2}} \\
p^{1 \mathrm{i}} & p^{1 \dot{2}} & 0 & 0 \\
p^{2 \mathrm{i}} & p^{2 \dot{2}} & 0 & 0
\end{array}\right)\left(\begin{array}{c}
\psi_{1 \mathrm{i}} \\
\psi_{1 \dot{2}} \\
\psi \\
0
\end{array}\right)=m\left(\begin{array}{c}
\psi_{1 \mathrm{i}} \\
\psi_{1 \dot{2}} \\
\psi \\
0
\end{array}\right), \\
& \left(\begin{array}{cccc}
0 & 0 & p_{1 \mathrm{i}} & p_{2 \mathrm{i}} \\
0 & 0 & p_{1 \dot{2}} & p_{2 \dot{2}} \\
p^{1 \mathrm{i}} & p^{1 \dot{2}} & 0 & 0 \\
p^{2 \mathrm{i}} & p^{2 \dot{2}} & 0 & 0
\end{array}\right)\left(\begin{array}{c}
\psi_{2 \mathrm{i}} \\
\psi_{2 \dot{2}} \\
0 \\
\psi
\end{array}\right)=m\left(\begin{array}{c}
\psi_{2 \mathrm{i}} \\
\psi_{2 \dot{2}} \\
0 \\
\psi
\end{array}\right),
\end{aligned}
$$

respectively, with one component equalling zero. Since in (13) and (14) there is the same differential operator we can write these equations as a single Dirac equation. Substituting explicit formulae for the spinors $p^{A \dot{B}}$ and $p_{A \dot{B}}$ (see [22]), we have

$$
\left(p^{0} \gamma^{0}-p^{1} \gamma^{1}-p^{2} \gamma^{2}-p^{3} \gamma^{3}\right) \mathbb{A}=m \mathbb{A},
$$

where $\mathbb{A}=\left(\begin{array}{ccc}\psi_{1 i} & \psi_{2 i} \\ \psi_{12} & \psi_{2 i} \\ \psi & 0 \\ 0 & \psi\end{array}\right)$ is a generalized (matrix) wavefunction and $\gamma^{\mu}$ matrices read

$$
\begin{aligned}
\gamma^{0} & =\left(\begin{array}{llll}
0 & 0 & 1 & 0 \\
0 & 0 & 0 & 1 \\
1 & 0 & 0 & 0 \\
0 & 1 & 0 & 0
\end{array}\right), \\
\gamma^{1} & =\left(\begin{array}{cccc}
0 & 0 & 0 & 1 \\
0 & 0 & 1 & 0 \\
0 & -1 & 0 & 0 \\
-1 & 0 & 0 & 0
\end{array}\right), \\
\gamma^{2} & =\left(\begin{array}{cccc}
0 & 0 & 0 & -i \\
0 & 0 & i & 0 \\
0 & i & 0 & 0 \\
-i & 0 & 0 & 0
\end{array}\right), \\
\gamma^{3} & =\left(\begin{array}{cccc}
0 & 0 & 1 & 0 \\
0 & 0 & 0 & -1 \\
-1 & 0 & 0 & 0 \\
0 & 1 & 0 & 0
\end{array}\right) .
\end{aligned}
$$


Let us note that this is the modified spinor representation with $\gamma^{i} \rightarrow-\gamma^{i}(i=1,2,3)$ and $\Psi=\left(\xi^{A}, \eta_{\dot{B}}\right)^{T} \rightarrow \Psi=$ $\left(\eta_{\dot{B}}, \xi^{A}\right)^{T}$ with respect to [28]. In what follows we will use a shorthand $\mathbb{A}=\left(\psi_{A \dot{B}}, \psi I_{2 \times 2}\right)^{T}$, where $I_{2 \times 2}$ is the $2 \times 2$ unit matrix.

3.2. Spin 1. We will now describe in two steps splitting of the DKP equations. To achieve first level of splitting we write DKP equations (7) in spinor notation as $[30,31]$

$$
\begin{aligned}
& p_{A}^{\dot{B}} \zeta_{C \dot{B}}+p_{C}^{\dot{B}} \zeta_{A \dot{B}}=2 m \eta_{A C}, \\
& p_{\dot{B}}^{A} \zeta_{A \dot{D}}+p_{\dot{D}}^{A} \zeta_{A \dot{B}}=2 m \chi_{\dot{B} \dot{D}} \\
& p_{A}^{\dot{C}} \chi_{\dot{B} \dot{C}}+p_{\dot{B}}^{C} \eta_{A C}=-2 m \zeta_{A \dot{B}} .
\end{aligned}
$$

Equations (17) are now splitted to yield two separate equations for spinors $\chi_{\dot{B} \dot{D}}$ and $\zeta_{A \dot{B}}$ and $\eta_{A C}$ and $\zeta_{A \dot{B}}$ :

$$
\begin{aligned}
& p_{A}^{\dot{B}} \zeta_{C \dot{B}}=m \eta_{A C}, \quad \eta_{A C}=\eta_{C A}, \\
& p_{\dot{B}}^{C} \eta_{A C}=-m \zeta_{A \dot{B}}, \\
& p_{\dot{B}}^{A} \zeta_{A \dot{D}}=m \chi_{\dot{B} \dot{D}}, \quad \chi_{\dot{B} \dot{D}}=\chi_{\dot{D} \dot{B}}, \\
& p_{A}^{\dot{D}} \chi_{\dot{B} \dot{D}}=-m \zeta_{A \dot{B}},
\end{aligned}
$$

respectively. The splitting was achieved due to appropriate spinor identities; see equation (11) in [22]. Indeed, solutions of (18) and (19) obey the DKP equations (17). This derivation was described in [22] and is included here for the sake of completeness.

Equations (18) and (19), first written by Dirac [32, 33], are known to describe spin 1 bosons where spinors $\eta_{C A}$ and $\chi_{\dot{D} \dot{B}}$ correspond to self-dual or anti-self-dual antisymmetric tensors $\psi^{\mu \nu}$, respectively [34]. Each of the above equations is covariant except from space reflection but (18) and (19) considered together are fully covariant. These equations written in tensor form, $\beta^{\mu} p_{\mu} \Psi=m \Psi, \Psi=$ $\left[\psi_{01}, \psi_{02}, \psi_{03}, \psi_{0}, \psi_{1}, \psi_{2}, \psi_{3}\right]^{T}$, where $\psi_{\mu \nu}$ are self-dual or antiself-dual antisymmetric tensors, with $7 \times 7$ matrices $\beta^{\mu}$ fulfilling the DKP algebra (4), are the Hagen-Hurley equations [35-38]. Explicit formulae for these $\beta^{\mu}$ matrices are given in [38].

We will now split the $s=1$ Dirac equations (18). Substituting expressions for $p_{A}^{\dot{B}}$ and $p_{\dot{B}}^{C}$ (cf. [22]), we obtain a system of eight equations:

$$
\begin{aligned}
-\left(p^{1}+i p^{2}\right) \zeta_{1 i}-\left(p^{0}-p^{3}\right) \zeta_{12} & =m \eta_{11} \\
\left(p^{0}+p^{3}\right) \zeta_{11}+\left(p^{1}-i p^{2}\right) \zeta_{12} & =m \eta_{21}
\end{aligned}
$$

$$
\begin{aligned}
&-\left(p^{1}-i p^{2}\right) \eta_{11}-\left(p^{0}-p^{3}\right) \eta_{12}=-m \zeta_{11}, \\
&\left(p^{0}+p^{3}\right) \eta_{11}+\left(p^{1}+i p^{2}\right) \eta_{12}=-m \zeta_{12}, \\
&-\left(p^{1}+i p^{2}\right) \zeta_{21}-\left(p^{0}-p^{3}\right) \zeta_{22}=m \eta_{12}, \\
&\left(p^{0}+p^{3}\right) \zeta_{21}+\left(p^{1}-i p^{2}\right) \zeta_{22}=m \eta_{22}, \\
&-\left(p^{1}-i p^{2}\right) \eta_{21}-\left(p^{0}-p^{3}\right) \eta_{22}=-m \zeta_{21}, \\
&\left(p^{0}+p^{3}\right) \eta_{21}+\left(p^{1}+i p^{2}\right) \eta_{22}=-m \zeta_{22},
\end{aligned}
$$

where all equations are arranged into two subsets (20a) and (20b) and we have not assumed yet that $\eta_{12}=\eta_{21}$.

Demanding now $\eta_{12}=\eta_{21} \equiv \eta$ we achieve splitting of (20a) and (20b) obtaining two Dirac-like equations:

$$
\left(p^{0} \widetilde{\gamma}^{0}-p^{1} \tilde{\gamma}^{1}-p^{2} \widetilde{\gamma}^{2}-p^{3} \widetilde{\gamma}^{3}\right) \Psi=m \Psi,
$$

with $\Psi=\left(\zeta_{11}, \zeta_{12}, \eta_{11}, \eta\right)^{T}$ and

$$
\left(p^{0} \widetilde{\gamma}^{0}-p^{1} \widetilde{\gamma}^{1}-p^{2} \widetilde{\gamma}^{2}-p^{3} \widetilde{\gamma}^{3}\right) \widetilde{\Psi}=m \widetilde{\Psi},
$$

with $\widetilde{\Psi}=\left(\zeta_{21}, \zeta_{2 \dot{2}}, \eta, \eta_{22}\right)^{T}$, where $\widetilde{\gamma}^{\mu}$ matrices are expressed by $\gamma^{\mu}$ matrices (16):

$$
\begin{aligned}
& \widetilde{\gamma}^{0}=i \gamma^{2}, \\
& \tilde{\gamma}^{1}=-\gamma^{3}, \\
& \tilde{\gamma}^{2}=i \gamma^{0}, \\
& \tilde{\gamma}^{3}=\gamma^{1} .
\end{aligned}
$$

The Dirac-like equations (21a) and (21b) are highly nonstandard because of several reasons: they contain higherorder spinors $\eta_{A B}$ and $\zeta_{A \dot{B}}$ and one common component $\left(\eta_{12}=\eta_{21} \equiv \eta\right)$ and their solutions, $\Psi$ and $\widetilde{\Psi}$, are not fully covariant since, considered separately, they do not involve all components of the spinors $\eta_{A B}$ and $\zeta_{A \dot{B}}$. On the other hand, these equations are fully covariant when considered as a whole.

Since in (21a) and (21b) there is the same differential operator we can write these equations as a single Dirac equation. We note, however, that the Dirac matrices in (16) and (22) are different. We thus first transform $\widetilde{\gamma}^{\mu}$ matrices unitarily to get $\gamma^{\mu}$ defined in (16):

$$
\begin{aligned}
\gamma^{\mu} & =V \tilde{\gamma}^{\mu} V^{\dagger}, \\
V & =\left(\begin{array}{cccc}
1 & 0 & 0 & 0 \\
0 & 1 & 0 & 0 \\
0 & 0 & 0 & 1 \\
0 & 0 & -1 & 0
\end{array}\right) .
\end{aligned}
$$

Now, we can write (21a) and (21b) as a single Dirac equation and with the same representation of $\gamma^{\mu}$ matrices as in (15):

$$
\left(p^{0} \gamma^{0}-p^{1} \gamma^{1}-p^{2} \gamma^{2}-p^{3} \gamma^{3}\right) \mathbb{B}=m \mathbb{B}
$$


where $\mathbb{B}=V\left(\begin{array}{cc}\zeta_{11} & \zeta_{21} \\ \zeta_{12} & \zeta_{22} \\ \eta_{11} & \eta \\ \eta & \eta_{22}\end{array}\right)=\left(\begin{array}{cc}\zeta_{11} & \zeta_{21} \\ \zeta_{1 \dot{2}} & \zeta_{2 \dot{2}} \\ -\eta_{1}^{1} & -\eta_{2}^{1} \\ -\eta_{1}^{2} & -\eta_{2}^{2}\end{array}\right)$ is a generalized matrix wavefunction. In what follows we will write $\mathbb{B}=\left(\zeta_{A \dot{B}},-\eta_{C}^{D}\right)^{T}$.

\section{Discussion}

We have demonstrated that, in the noninteracting case, full covariant solutions of the $s=0$ and $s=1$ DKP equations are generalized solutions of the same Dirac equation; see (1), (15), and (24). More exactly, if we choose the modified spinor representation of the Dirac matrices defined in (16) then the following functions $\Psi=\left(\eta_{\dot{B}}, \xi^{A}\right)^{T}, \mathbb{A}=\left(\psi_{A \dot{B}}, \psi I_{2 \times 2}\right)^{T}$, and $\mathbb{B}=\left(\zeta_{A \dot{B}},-\eta_{C}^{D}\right)^{T}$ with $\eta_{C D}=\eta_{D C}$ are solutions of the same Dirac equation and correspond to $s=1 / 2, s=0$, and $s=$ 1 cases, respectively. We note that $\mathbb{A}$ and $\mathbb{B}$ are generalized (matrix) solutions. It follows that these solutions of the Dirac equation provide synthesis of relativistic equations for $s=1 / 2$ and $s=0,1$.

Similar generalized solutions exist also in the interacting case. Indeed, although, in the $s=0$ case, (3) and (15) are not equivalent in general fields, they are equivalent in crossed fields [39]. Similarly, the Dirac equations (18) or (19) are not equivalent to the $s=1 \mathrm{DKP}$ equations (17) in general fields. It remains to determine if the $s=1$ Dirac equations are equivalent to the $s=1 \mathrm{DKP}$ equations in some special fields.

\section{Conflict of Interests}

The author declares that there is no conflict of interests regarding the publication of this paper.

\section{References}

[1] N. Kemmer, "The particle aspect of meson theory," Proceedings of the Royal Society of London Series A: Mathematical and Physical Sciences, vol. 173, no. 952, pp. 91-116, 1939.

[2] R. J. Duffin, "On the characteristic matrices of covariant systems," Physical Review, vol. 54, no. 12, p. 1114, 1938.

[3] G. Petiau, "University of Paris thesis," Académie Royale de Médecine de Belgique, Classe des Sciences, Mémoire, vol. 8, no. 16, p. 2, 1936.

[4] H. Hassanabadi, B. H. Yazarloo, S. Zarrinkamar, and A. A. Rajabi, "Duffin-Kemmer-Petiau equation under a scalar Coulomb interaction," Physical Review C: Nuclear Physics, vol. 84, no. 6, Article ID 064003, 2011.

[5] Y. Kasri and L. Chetouani, "Energy spectrum of the relativistic Duffin-Kemmer-Petiau equation," International Journal of Theoretical Physics, vol. 47, no. 9, pp. 2249-2258, 2008.

[6] Y. Chargui, A. Trabelsi, and L. Chetouani, "Bound-states of the $(1+1)$-dimensional DKP equation with a pseudoscalar linear plus Coulomb-like potential," Physics Letters A, vol. 374, no. 29, pp. 2907-2913, 2010.

[7] M. Hamzavi and S. M. Ikhdair, "Any J-state solution of the Duffin-Kemmer-Petiau equation for a vector deformed woodssaxon potential," Few-Body Systems, vol. 53, no. 3-4, pp. 461-471, 2012.

[8] F. Yaşuk, C. Berkdemir, A. Berkdemir, and C. Önem, "Exact solutions of the Duffin-Kemmer-Petiau equation for the deformed hulthen potential," Physica Scripta, vol. 71, no. 4, pp. 340-343, 2005.

[9] H. Hassanabadi, Z. Molaee, and S. Zarrinkamar, "DKP oscillator in the presence of magnetic field in $(1+2)$-dimensions for spin-zero and spin-one particles in noncommutative phase space," European Physical Journal C, vol. 72, article 2217, 2012.

[10] A. Boumali and H. Hassanabadi, "The thermal properties of a two-dimensional Dirac oscillator under an external magnetic field," The European Physical Journal Plus, vol. 128, no. 10, article 124, 2013.

[11] H. Hassanabadi, S. S. Hosseini, A. Boumali, and S. Zarrinkamar, "The statistical properties of Klein-Gordon oscillator in noncommutative space," Journal of Mathematical Physics, vol. 55, no. 3, Article ID 033502, 2014.

[12] M. Darroodi, H. Hassanabadi, and N. Salehi, "The modified Woods-Saxon potential in the Duffin-Kemmer-Petiau equation," The European Physical Journal A, vol. 51, no. 6, article 69, 2015.

[13] N. Salehi and H. Hassanabadi, "Scattering amplitude of the Duffin-Kemmer-Petiau equation for the Yukawa potential for $\mathrm{J}=0$," The European Physical Journal A, vol. 51, no. 6, article 100, 2015.

[14] V. I. Fushchich and A. G. Nikitin, "Poincare invariant equations of motion for particles of arbitrary spin," Soviet Journal of Particles and Nuclei, vol. 9, p. 501, 1978.

[15] W. I. Fushchich and A. G. Nikitin, Symmetries of Equations of Quantum Mechanics, Allerton Press Inc, New York, NY, USA, 1994.

[16] G. Velo and D. Zwanzinger, "Noncausality and other defects of interaction lagrangians for particles with spin one and higher," Physical Review, vol. 188, no. 5, pp. 2218-2222, 1969.

[17] A. S. Wightman, "Relativistic wave equations as singular hyperbolic systems," in Partial Differential Equations, D. C. Spencer, Ed., vol. 23, pp. 441-477, American Mathematical Society, Providence, RI, USA, 1973.

[18] K. Johnson and E. C. G. Sudarshan, "Inconsistency of the local field theory of charged spin 3/2 particles," Annals of Physics, vol. 13 , no. 1, pp. 126-145, 1961.

[19] A. S. Wightman, "The stability of representations of the Poincaré group," in Proceedings of the 5th Coral Gables Conference on Symmetry Principles at High Energies, A. Perlmutter, C. A. Hurst, and B. Kursonoglu, Eds., University of Miami, Coral Gables, Fla, USA, 1968.

[20] G. Velo and D. Zwanziger, "Propagation and quantization of Rarita-Schwinger waves in an external electromagnetic potential," Physical Review, vol. 186, no. 5, pp. 1337-1341, 1969.

[21] A. Okninski, "Supersymmetric content of the Dirac and DuffinKemmer-Petiau equations," International Journal of Theoretical Physics, vol. 50, no. 3, pp. 729-736, 2011.

[22] A. Okniński, "Splitting the Kemmer-Duffin-Petiau equations," in Proceedings of Institute of Mathematics of NAS of Ukraine, vol. 50, part 2, pp. 902-908, 2004.

[23] A. Okniński, "On the mechanism of fermion-boson transformation," International Journal of Theoretical Physics, vol. 53, no. 8, pp. 2662-2667, 2014.

[24] A. Okniński, "Neutrino-assisted fermion-boson transitions," Acta Physica Polonica B, vol. 46, no. 2, pp. 221-229, 2015.

[25] L. L. Foldy, "Synthesis of covariant particle equations," Physical Review, vol. 102, no. 2, pp. 568-581, 1956.

[26] P. A. M. Dirac, "The quantum theory of the electron," Proceedings of the Royal Society of London A, vol. 117, no. 778, pp. 610624, 1928. 
[27] P. A. M. Dirac, "The quantum theory of the electron. Part II," Proceedings of the Royal Society of London Series A: Containing Papers of a Mathematical and Physical Character, vol. 118, no. 779, pp. 351-361, 1928.

[28] V. B. Berestetskii, E. M. Lifshits, and L. P. Pitaevskii, Relativistic Quantum Theory, Pergamon Press, 1974.

[29] A. Proca, "Sur les equations fondamentales des particules elementaires," Comptes Rendus de l'Académie des Sciences de Paris, vol. 202, pp. 1490-1492, 1936.

[30] J. Łopuszański, Spinor Calculus, PWN Polish Scientific Publishers, Warsaw, Poland, 1985 (Polish).

[31] A. H. Taub, "Spinor equations for the meson and their solution when no field is present," Physical Review, vol. 56, no. 8, pp. 799810, 1939.

[32] E. M. Corson, Introduction to Tensors, Spinors, and Relativistic Wave Equations, Blackie and Son, London, UK, 1953.

[33] P. A. M. Dirac, "Relativistic wave equations," Proceedings of the Royal Society of London A, vol. 155, no. 886, pp. 447-459, 1936.

[34] J. T. Łopuszański, "The representations of the Poincaré group in the framework of free quantum fields," Fortschritte der Physik, vol. 26, no. 4, pp. 261-288, 1978.

[35] C. R. Hagen and W. J. Hurley, "Magnetic moment of a particle with arbitrary spin," Physical Review Letters, vol. 24, no. 24, pp. 1381-1384, 1970.

[36] W. J. Hurley, "Relativistic wave equations for particles with arbitrary spin," Physical Review D, vol. 4, no. 12, pp. 3605-3616, 1971.

[37] W. J. Hurley, "Invariant bilinear forms and the discrete symmetries for relativistic arbitrary-spin fields," Physical Review D, vol. 10, no. 4, pp. 1185-1200, 1974.

[38] J. Beckers, N. Debergh, and A. G. Nikitin, "On parasupersymmetries and relativistic descriptions for spin one particles: I. The free context," Fortschritte der Physik, vol. 43, no. 1, pp. 67-80, 1995.

[39] A. Okniński, "Duffin-Kemmer-Petiau and Dirac equations-a supersymmetric connection," Symmetry, vol. 4, no. 3, pp. 427440, 2012. 


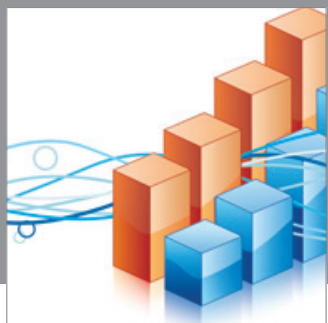

Advances in

Operations Research

mansans

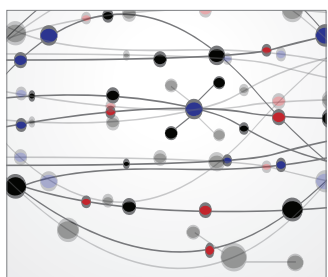

The Scientific World Journal
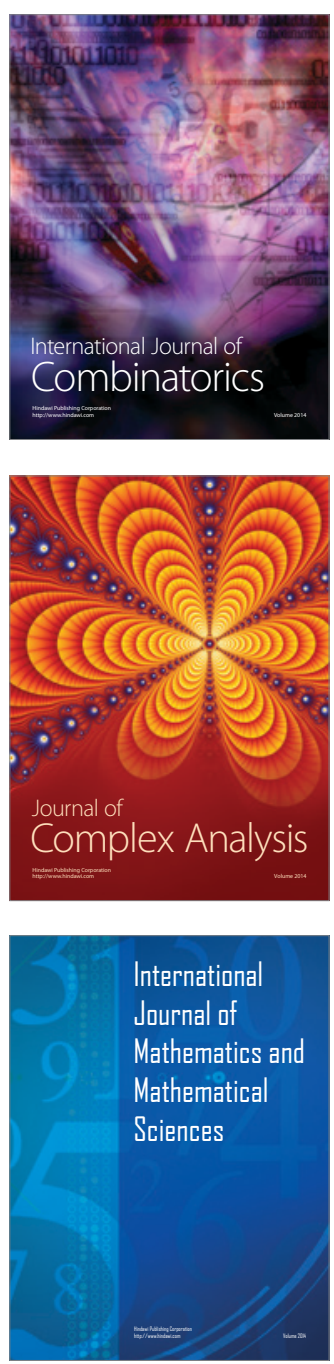
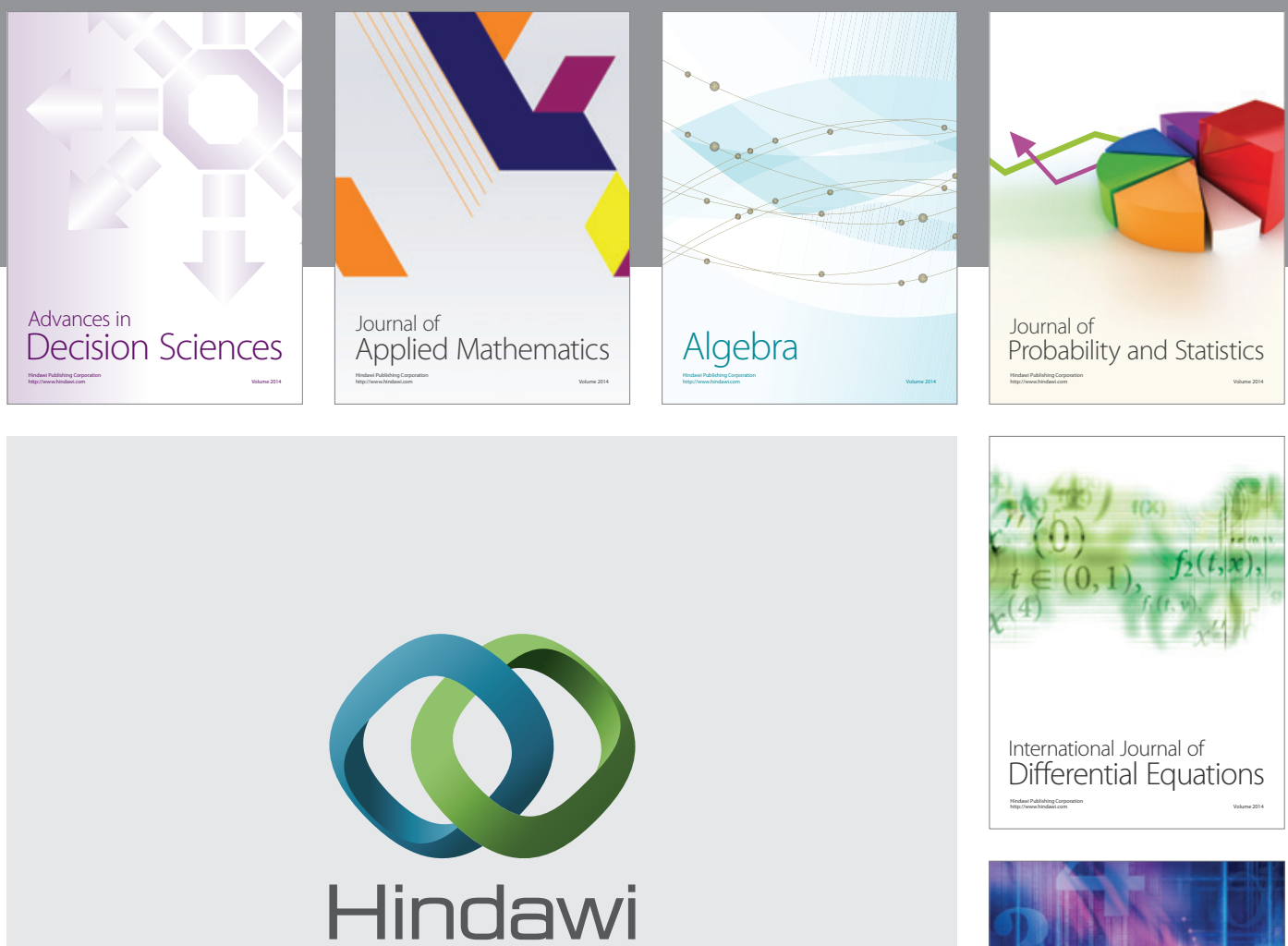

Submit your manuscripts at http://www.hindawi.com
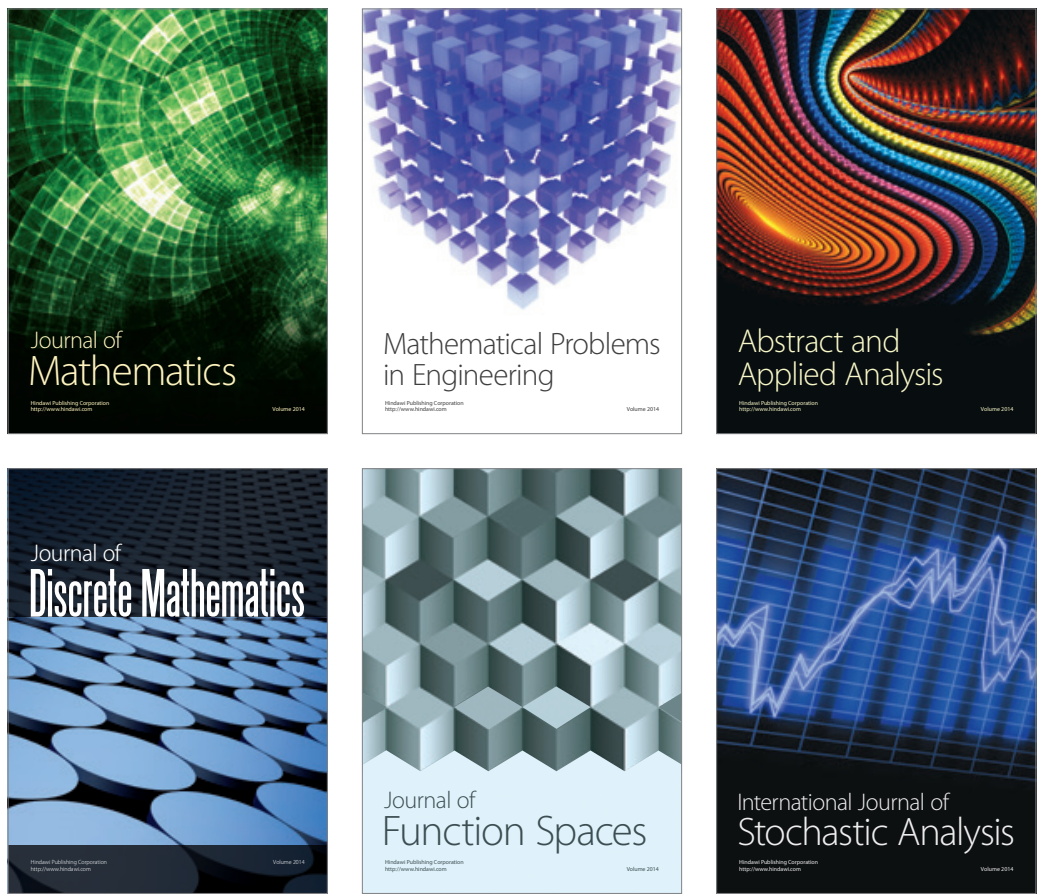

Journal of

Function Spaces

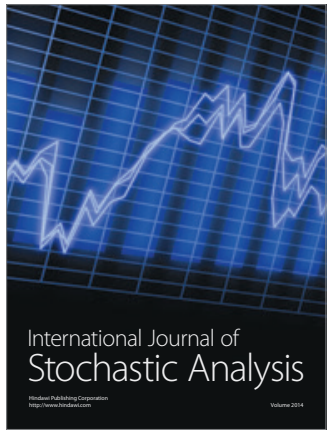

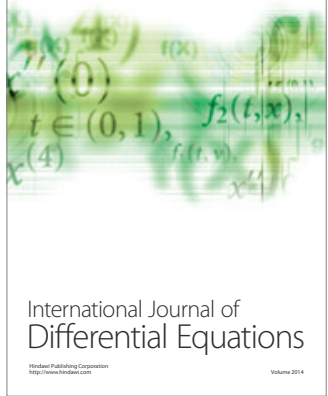
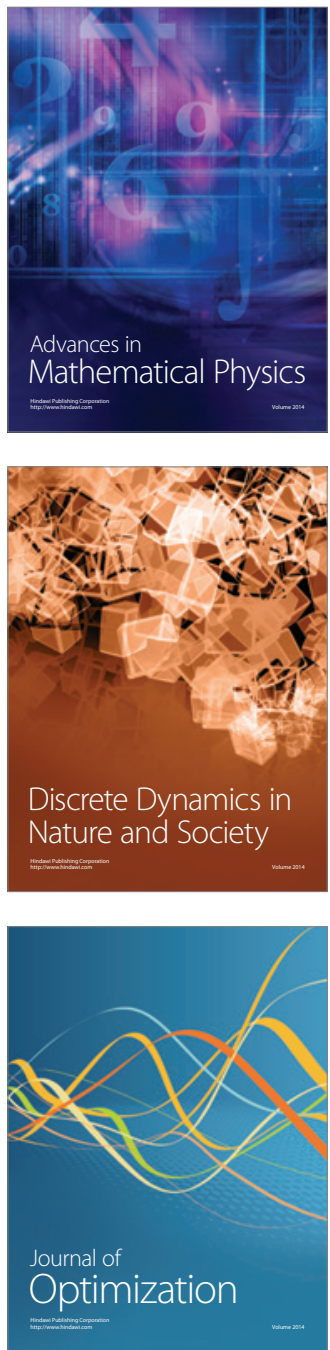